\title{
Nonequilibrium Thermodynamics and Distributions Time to Achieve a Given Level of a Stochastic Process for Energy of System
}

\author{
V. V. Ryazanov \\ Institute for Nuclear Research, Prospect Nauki 47, Kiev 252028, Ukraine \\ Correspondence should be addressed to V. V. Ryazanov, vryazan@kinr.kiev.ua
}

Received 21 August 2012; Revised 9 October 2012; Accepted 9 October 2012

Academic Editor: L. De Goey

Copyright $\odot 2012$ V. V. Ryazanov. This is an open access article distributed under the Creative Commons Attribution License, which permits unrestricted use, distribution, and reproduction in any medium, provided the original work is properly cited.

In a previous paper (Ryazanov (2011)) with the joint statistical distribution for the energy and lifetime (time to achieve a given level of a stochastic process for energy of system) to derive thermodynamic relationships, clarifying similar expressions of extended irreversible thermodynamics we used an exponential distribution of lifetime. In this paper, we explore a more realistic expression for the distribution of time to achieve a given level of a stochastic process for energy of system (or relaxation times or lifetimes), and we analyse how such distribution affects the corresponding expressions of nonequilibrium entropy, temperature, and entropy production.

\section{Introduction}

In [1] expressions for a nonequilibrium entropy $S$, entropy production $\sigma$, nonequilibrium temperature, and generalized transport equations for thermodynamic fluxes were proposed, taking into account a given distribution of internal relaxation times (or lifetimes) of the variables of the system. In the theory of random processes such values are called time to achieve a given level of the variables of the system (or the first-passage-time problems or escape time). In [1] for this quantity we used the term lifetime. These expressions generalize similar expressions of the extended irreversible thermodynamics (EIT) [2], a theory which takes into account the nonvanishing character of the relaxation time of the heat flux and other thermodynamic fluxes. In [1] we proposed to take into consideration not only a single or a few relaxation time, but a whole distribution of relaxation times, generalizing in this way the previous proposals of EIT.

There are many motivations for taking a full distribution of relaxation times; for instance, in heat transfer, the heat flux is the sum of contributions of molecules moving at different speeds, or of phonons having different frequencies, and the relaxation times of the mentioned contributions usually depend on the speed or the frequency, thus yielding a relaxation time distribution. Glassy materials have also a wide distribution of relaxation times. Collisions of heavy nuclei yield different products having widely different decay times. Thus, the consideration of relaxation time distributions seems natural in the analysis of nonequilibrium systems.

Approach [1] was based on the distribution containing lifetime as a thermodynamic parameter of the form

$$
\rho(z ; E=v, \Gamma=y)=\exp \frac{\{-\beta v-\gamma y\}}{Z(\beta, \gamma)},
$$

where $E$ is energy of the system, $z$ are dynamical variables, coordinates and momenta of the system particles, $\Gamma$ is random variables of lifetime, a first-passage time till the random process $v(t)=E(t)$ reaches its zero value, $\beta$ and $\gamma$ are Lagrange multipliers conjugated to thermodynamic parameters $E$ and $\Gamma$, and $Z(\beta, \gamma)$ is the partition function. Reference [1] uses an exponential distribution for the lifetime

$$
p_{\Gamma}(y)=\Gamma_{0}^{-1} \exp \left\{-\frac{y}{\Gamma_{0}}\right\},
$$

where $\Gamma_{0}$ is average lifetime. The expression (2) can be obtained from the algorithm of phase coarsening of the complex systems (Appendix 2 [1]). The distribution in the form (2) is valid for the existence of the weak ergodicity in a system. Mixing the system states at long times will lead 
to the distribution (2). For systems in the vicinity of phase transitions or in the chaotic regime, distribution (2) is no longer valid. The aim of this paper is to generalize the formalism proposed in [1] to this more general situation.

\section{Generalized Lifetime Thermodynamics with Lifetime Distribution in (3)}

Setting the form of the function $p_{\Gamma}(y)$ reflects not only the internal properties of a system, but also the influence of the environment on an open system and the particular character of its interaction with the environment. The following physical interpretation of the exponential distribution for the function $p_{\Gamma}(y)$ is given: a system evolves freely like an isolated system governed by the Liouville operator. Besides that the system undergoes random transitions and the phase point representing the system switches from one trajectory to another one with an exponential probability under the influence of the "thermostat." The exponential distribution describes completely random systems. The influence of the environment on a system can have organized character as well; for example, this is the case of systems in a nonequilibrium state with input and output nonstationary fluxes. The character of the interaction with the environment can also vary; therefore different forms of the function $p_{\Gamma}(y)$ can be used.

In [3] the maximum entropy principle for Liouville equations with source for the determination of the function $p_{\Gamma}(y)$ was applied. The result obtained in [3] can be written as

$$
\begin{array}{r}
p_{\Gamma}(y, t)=\frac{p_{\Gamma}(0) e^{-C_{i} y / F_{i}}}{1+\left(p_{\Gamma}(0) / F_{i}\right) e^{-C_{i} y / F_{i}}\left(R(t)-R\left(t_{0}\right)\right)}, \\
y=t-t_{0},
\end{array}
$$

where $t_{0}$ is some initial moment of birth of the system, $t$ is the current time,

$$
\begin{aligned}
R(t)= & \sum_{j} \sum_{m} F_{m}\left(t_{0}\right) F_{j}\left(t_{0}\right) \\
& \times \sum_{k} \frac{\left\langle P_{k} P_{j} P_{m}\right\rangle-\left\langle P_{j} P_{m}\right\rangle\left\langle P_{k}\right\rangle}{\left\langle P_{i} P_{k}\right\rangle-\left\langle P_{i}\right\rangle\left\langle P_{k}\right\rangle}+F_{i} \ln Z_{\beta}\left(t_{0}\right)- \\
& -\sum_{m} \sum_{j} F_{j}\left(t_{0}\right) \frac{\left\langle P_{j} P_{m}\right\rangle-\left\langle P_{j}\right\rangle\left\langle P_{m}\right\rangle}{\left\langle P_{i} P_{m}\right\rangle-\left\langle P_{i}\right\rangle\left\langle P_{m}\right\rangle} .
\end{aligned}
$$

In (4) it is assumed that, along with the energy $E=$ $P_{0}$ system is described and by $M$ other physical quantities; for example, $P_{1}=p$ is density of momentum, $P_{i+1}=n_{i}$ are the number of particles ith component, and so forth and conjugate thermodynamic parameters $F_{j}$. If we restrict ourselves, $P_{0}=E, F_{j}=\beta, R(t)$ becomes

$$
\begin{aligned}
R(t)= & \beta^{2}\left(t_{0}\right) \frac{\left\langle E^{3}\right\rangle-\left\langle E^{2}\right\rangle\langle E\rangle}{\left\langle E^{2}\right\rangle-\langle E\rangle^{2}}+\beta(t) \ln Z_{\beta}\left(t_{0}\right) \\
& -\beta\left(t_{0}\right), \quad Z_{\beta}=\int \omega(E=v) e^{-\beta v} d v .
\end{aligned}
$$
$[4,5]$

In deriving (3) we used the Zubarev-Peletminsky rule

$$
\begin{gathered}
\frac{d \vec{z}}{d t}=\vec{w}(\vec{z}), \\
\vec{w} \vec{\nabla} P_{i}=\sum_{j=1}^{M} C_{i j} P_{j} ; \quad i=1, \ldots, M, \\
C_{i}=\sum_{j} C_{j i} F_{j}\left(t_{0}\right),
\end{gathered}
$$

where $C_{i j}$ are $c$-numbers. When the local density of dynamic variables is considered, the value of $P_{m}$ may depend on the spatial variables. Since then the value $C_{i j}$ may also depend on the spatial variables or may be differential operators. The equation for the specific energy $u$ has the form $\rho d u / d t+$ $\vec{\nabla} \vec{J} q=0 ; E=\int_{V} \rho u d V ; \overrightarrow{J_{q}}=\overrightarrow{J_{u}}=\vec{q}$ is the heat flux. In this paper we consider what changes in the thermodynamic behavior of the system results with replacement of the form (2), used in [1], the function of the form (3). that

From the normalization of the distribution (3) it is seen

$$
\begin{aligned}
p_{\Gamma}(0) & =\frac{\beta\left(1-e^{-r C_{i} / \beta^{2}}\right)}{r}, \\
r & =R\left(t_{0}\right)-R(t) .
\end{aligned}
$$

The value of $\left(C_{i} / \beta\right)^{-1}$ is close to the average lifetime $\Gamma_{0}$ of the relation (2). It may be shown that in the linear approximation to $r$

$$
\begin{gathered}
p_{\Gamma}\left(0, t_{0}\right)=a=\frac{C_{i}}{F_{i}}, \\
p_{\Gamma}(y, t)=a e^{-a y}\left(1+\frac{a r e^{-a y}}{\beta}\right) .
\end{gathered}
$$

In the expression for the partition function

$$
\begin{aligned}
Z(\beta, \gamma) & =\int \exp \{-\beta v-\gamma y\} d z \\
& =\iint d v d y \omega(v, y) \exp \{-\beta v-\gamma y\}
\end{aligned}
$$

is the structure factor $\omega(E, \Gamma)$ which has a meaning of the joint probability density of values $E, \Gamma$ as it was shown in [1]. It may be shown that for the distribution (3) the function $\omega(E, \Gamma)$ takes on the form:

$$
\begin{aligned}
\omega(E, \Gamma & =y) \\
& =\omega(E) \frac{p_{\Gamma}(0) e^{-\left(C_{i} y / F_{i}\right)}}{1+\left(p_{\Gamma}(0) / F_{i}\right) e^{-\left(C_{i} y / F_{i}\right)}\left(R(t)-R\left(t_{0}\right)\right)} .
\end{aligned}
$$


Substituting (10) into the partition function (9) yields

$$
\begin{aligned}
Z(\beta, \gamma)= & Z(\beta) Z(\gamma), \\
Z(\gamma)= & \int_{0}^{\infty} e^{-\gamma y} p_{\Gamma}(y, t) d y \\
& =\frac{p_{\Gamma}(0)}{a(1+(\gamma / a))}{ }_{2} F_{1}\left(1,1+\frac{\gamma}{a}, 2+\frac{\gamma}{a}, w\right), \\
& w=\frac{p_{\Gamma}(0) r}{\beta},
\end{aligned}
$$

where $Z(\beta)=\int \omega(E=v) \exp \{-\beta v\} d v$ is the Gibbs partition function, ${ }_{2} F_{1}(., ., .,$.$) is ordinary hypergeometric function$ [6].

We have from (11) when $\Gamma \gamma=-\partial \ln Z(\beta, \gamma) / \partial \gamma$; $\Gamma_{0}(V)=\Gamma_{\gamma}(V) / \gamma=0$;

$$
\begin{gathered}
\gamma \Gamma \gamma=\frac{x}{1+x}-x \frac{w\left(1 /(2+x)^{2}\right)+\cdots+w^{n}\left(n /(x+n+1)^{2}\right)+\cdots}{1+w((x+1) /(x+2))+\cdots+w^{n}((x+1) /(x+n+1))+\cdots}, \quad x=\frac{\gamma}{a} \approx \gamma \Gamma_{0}, \\
{ }_{2} F_{1}(1, x+1, x+2, w)=1+w \frac{x+1}{x+2}+\cdots+w^{n} \frac{x+1}{x+n+1}+\cdots
\end{gathered}
$$

The expression for $x=\gamma / a$ is close to that considered in [1], the expression $x=\gamma \Gamma_{0}$, but not identical to it. At $Z(\beta, \gamma)=$ $Z(\beta) Z(\gamma)$ the entropy is equal to

$$
\begin{aligned}
\frac{S}{k_{B}} & =-\langle\ln \rho(z ; E, \Gamma)\rangle=\frac{S_{\beta}}{k_{B}}+\frac{S_{\gamma}}{k_{B}}, \quad(13) \\
S_{\beta}= & \beta\langle E\rangle+\ln Z(\beta), \quad S_{\gamma}=\gamma\langle\Gamma\rangle+\ln Z(\gamma), \\
S_{\gamma}= & \frac{x}{1+x}-\ln (1+x)+\ln \frac{p_{\Gamma}(0)}{a}-x \frac{w\left(1 /(2+x)^{2}\right)+\cdots+w^{n}\left(n /(x+n+1)^{2}\right)+\cdots}{1+w((1+x) /(2+x))+\cdots+w^{n}((1+x) /(1+n+x))+\cdots}+ \\
& +\ln \left[1+w \frac{(1+x)}{(2+x)}+\cdots+w^{n} \frac{(1+x)}{(1+n+x)}+\cdots\right] .
\end{aligned}
$$

The first two terms on the right side of (14) coincide with

From (14) the expression for $S_{\gamma}$ of [1]. The remaining terms are a supplement to this expression. They depend on $w$ and tend to 0 if a value $w$ (or a value $r$ in (11), (3), (7)) tends to 0 .

From (14) treating $E$ and $\Gamma$ as variables,

$$
d S=k_{B} \beta d E+k_{B} \gamma d \Gamma
$$

$$
\begin{aligned}
& \frac{\partial S_{y}}{\partial x}=-\frac{D}{x}, \\
& D=x\left\{\left(\frac{x}{(1+x)^{2}}\right)-\frac{x}{1+w((1+x) /(2+x))+\cdots+w^{n}((1+x) /(1+n+x))+\cdots}\right. \\
& \quad+\left[2\left(\frac{w}{(2+x)^{3}}+\cdots+\frac{w^{n} n}{(1+n+x)^{3}}+\cdots\right)+\right. \\
&\left.\left.\quad+\frac{\left(\left(w /(2+x)^{2}\right)+\cdots+\left(w^{n} n /(1+n+x)^{2}\right)+\cdots\right)^{2}}{1+w((1+x) /(2+x))+\cdots+w^{n}((1+x) /(1+n+x))+\cdots}\right]\right\} .
\end{aligned}
$$


In [1] for the case of thermal conductivity obtained the expression

$$
\begin{array}{r}
x_{q}=\gamma_{q} \Gamma_{0} \approx \frac{t_{0 q} S_{a} q}{E}=\frac{t_{0 q} q}{\rho u L}=t_{0 q} y_{q}, \\
y_{q}=\frac{q}{\rho u L}, E \approx \rho u V,
\end{array}
$$

where $q= \pm|\vec{q}|=(\vec{q} \vec{q})^{1 / 2}, u=E / \rho V$ is energy density in a system, $L$ is its linear size, $V$ is its volume $\sim L^{3}$, and the surface $S_{a} \sim L^{2} ; q_{+}=\left(\vec{q}_{+} \vec{s}\right)=q=(\vec{q} \vec{q})^{1 / 2}$, where $q_{-}$and $q_{+}$are projections of the outcoming and incoming heat flux density vectors on the surface normal vector $\vec{s}$ (ordered chronologically [1]); the signs of $q_{-}$and $q_{+}$depend on whether we are heating or cooling the system. Then $q_{+}=$ $-q_{-}-R \rho \partial u / \partial t$; in the stationary state $q_{+}=-q_{-}$.

Thermodynamic parameters that determine the value of $x$ will be the energy $E$, the heat flux $q$, and the size of $L$. From (17) we obtain

$$
d x=x\left(-\frac{d E}{E}+\frac{d q}{q}+\frac{2 d L}{L}\right)
$$

Expression (15) can be rewritten as

$$
d S=k_{B} \beta d E+\frac{\partial S_{\gamma}}{\partial x} d x
$$

The value $r$ in (11) is small, although it is possible to specify a physical situation where the value of $r$ is not small. In the linear approximation to $r$ (and $w$ ) the relationship (16) takes the form

$$
\frac{\partial S_{\gamma}}{\partial x} \approx-\frac{x}{(1+x)^{2}}+w \frac{2 x}{(2+x)^{3}} .
$$

In [1] value of $x$ was determined by comparing the obtained expression for the nonequilibrium entropy to the same expression of the EIT [2]. It is possible to determine the value of $x$ in the other ratios, for example, by comparing the expression for the inverse nonequilibrium temperature with corresponding expression of EIT in the form

$$
\theta^{-1}=\frac{1}{T}+q^{2} \frac{\alpha_{q}}{u}, \quad \alpha_{q}=\frac{\tau_{q}}{\rho \lambda T^{2}},
$$

where $\tau_{q}$ is the time of the flux correlation, $T$ is equilibrium temperature, and $\lambda$ is the heat conductivity coefficient.

The expression of $\theta^{-1}=\left(\partial S / \partial E_{\mid q, R}\right)$, obtained from (14), (16), (18) in the linear approximation to $w$ is given by

$$
\begin{aligned}
\theta^{-1} & =\frac{\partial S}{\partial E}_{\mid q, R} \\
& =k_{B}\left[\beta+\frac{x}{E}\left(\frac{x}{(1+x)^{2}}-w \frac{2 x}{(2+x)^{3}}\right)\right] .
\end{aligned}
$$

Equating the expressions (21) and (22), we obtain the equation for $x$. Neglecting the powers of $x$ higher than the second, we find the solution of the resulting quadratic equation

$$
x=\frac{-28 c \pm(16 c(30 c+4-w))^{1 / 2}}{2(38 c-8-2 w)}, \quad c=\frac{q^{2} \alpha_{q} E}{u k_{B}} .
$$

The exact expression of $\theta^{-1}$ is more cumbersome ((20) than linear approximation of (16))

$$
\theta^{-1}=k_{B}\left(\beta+\frac{D}{E}\right)
$$

where $D$ is from (16). Here you can substitute the value of $x$ from (17). From (16)-(19) we obtain

$$
\begin{aligned}
d S= & k_{B}\left(\beta+\frac{D}{E}\right) d E \\
& -k_{B} \frac{D}{q} d q-k_{B} \frac{2 D}{L} d L .
\end{aligned}
$$

Determining in (25) the value of $d S / d t$, we find the entropy balance equation $(1 / V)(d S / d t)=-\vec{\nabla} \overrightarrow{j_{S}}+\sigma_{S}, S=$ $\int_{V} \rho s d \vec{r}$, where for $\rho=$ const, $\overrightarrow{j_{S}}=\theta^{-1} \vec{q}$,

$$
\begin{aligned}
\sigma_{S}= & \vec{q} \vec{\nabla} \theta^{-1}-\frac{k_{B} D(d q / d t)}{q V} \\
& +\frac{k_{B}(d L / d t)\left[3 u \beta \rho+V^{-1} D\right]}{L} .
\end{aligned}
$$

Comparing entropy production $\sigma_{S}(26)$ and $\sigma_{S}=\vec{q} \vec{q} / \lambda T^{2}$ of EIT [2], we shall find

$$
\begin{aligned}
\lambda T^{2} k_{B}\left(\frac{d \vec{q}}{d t}\right) q^{-2} V^{-1} D+\vec{q} \\
=\lambda T^{2} \nabla \theta^{-1}+ \\
+\left(\frac{d L}{d t}\right) \lambda T^{2} k_{B} \frac{\left[3 u \beta \rho+D V^{-1}\right]}{\vec{q} L} .
\end{aligned}
$$

The first two terms of the expressions (24)-(27) coincide with the expressions of [1]. The remaining terms are a supplement to these expressions. They depend on $w$ and tends to 0 if a value $w$ tends to 0 .

Just as in [1], we can write the conditions of thermodynamic stability and also consider not only by heat transfer, but by the internal friction of the system which is represented by the dissipative part of the stress tensor, the processes with variable mass, and so forth.

\section{Conclusion}

In this paper instead of the exponential distribution for the lifetime of [1] we examined the lifetime distribution of the form (3). The expressions derived here generalize expressions for the nonequilibrium entropy, entropy production, inverse nonequilibrium temperature, and Maxwell-Cattaneo equation derived in [1]. Expressions have the following structure: the terms of [1] and supplements, depending on the value of $w(11)$. 
There are other possibilities to generalize the results of [1]. Thus, in [7] the linearised dual-phase-lag model of heat transfer is based on the equation

$$
\tau \frac{\partial q}{\partial t}+q=-\lambda \nabla T-\varepsilon \frac{\partial \nabla T}{\partial t}
$$

where $T$ is the equilibrium temperature, $\lambda$ the heat conductivity, $q$ the heat flux, $\tau$ and $\varepsilon$ are relaxation times. In [7] it was supposed that the entropy density $s$ depends not only on the internal energy density $u$ but also on $\dot{u}$ and $\ddot{u}=\left(\partial^{2} u\right) /\left(\partial t^{2}\right), s=s(u, \dot{u}, \ddot{u})$. The use of these variables could be extended to higher-order time derivatives; such formalism is compatible with our proposal of taking a distribution of relaxation times, because it allows for rather general dynamical decays, much more complicated than an exponential decay with a single relaxation time.

It is possible to suggest some actual physical systems to which these ideas may be applicable. Those are systems which take into account the nonvanishing character of the relaxation time of the heat flux and other thermodynamic fluxes. Examples include solids with phonons having different frequencies and the relaxation times of the mentioned contributions usually depend on the speed or the frequency. A more detailed study of glass materials with a wide range of relaxation times, different times of the decay in collisions of heavy nuclei-those and other examples-can serve as objects of description of the proposed theory. The proposed description also applies to the processes of deformation of a continuous medium, to the chemical reactions, and so forth. The proposed description characterizes open systems describing the interaction with the environment.

\section{References}

[1] V. V. Ryazanov, "Nonequilibrium thermodynamics based on the distributions containing lifetime as thermodynamic parameter," Journal of Thermodynamics, vol. 2011, Article ID 203203, 10 pages, 2011.

[2] D. Jou, J. Casas-Vazquez, and G. Lebon, Extended Irreversible Thermodynamics, Springer, Berlin, Germany, 1993.

[3] V. V. Ryazanov, "Maximum entropy principle and the form of source in non-equilibrium statistical operator method," http://arxiv.org/abs/0910.4490v1.

[4] D. N. Zubarev, Nonequilibrium Statistical Thermodynamics, Plenum-Consultants Bureau, New York, NY, USA, 1974.

[5] A. I. Akhiezer and S. V. Peletminskii, Methods of Statistical Physics, Pergamon Press, Oxford, UK, 1981.

[6] M. Abramowitz and I. A. Stegun, Eds., Handbook of Mathematical Functions with Formulas, Graphs, and Mathematical Tables, Dover, New York, NY, USA, 1972.

[7] S. I. Serdyukov, "A new version of extended irreversible thermodynamics and dual-phase-lag model in heat transfer," Physics Letters A, vol. 281, no. 1, pp. 16-20, 2001. 

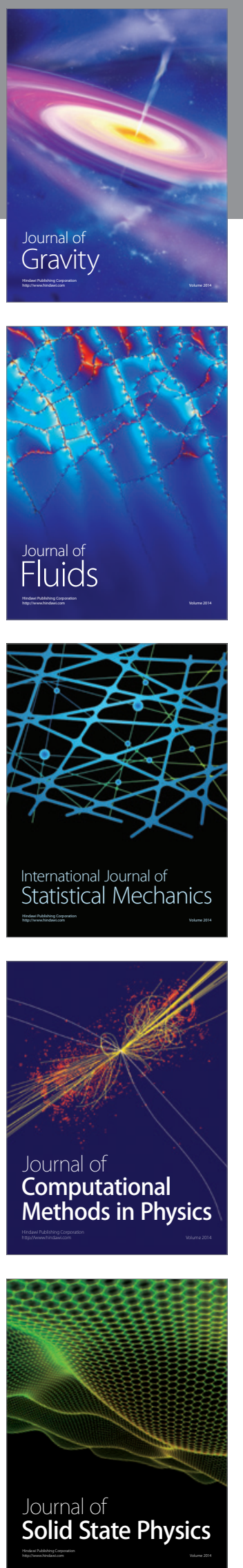

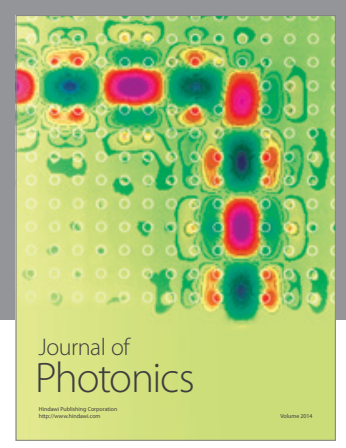

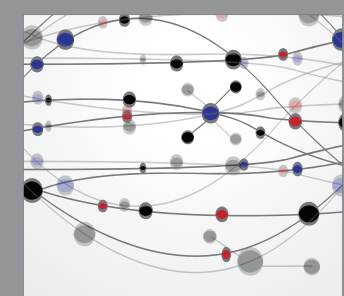

The Scientific World Journal
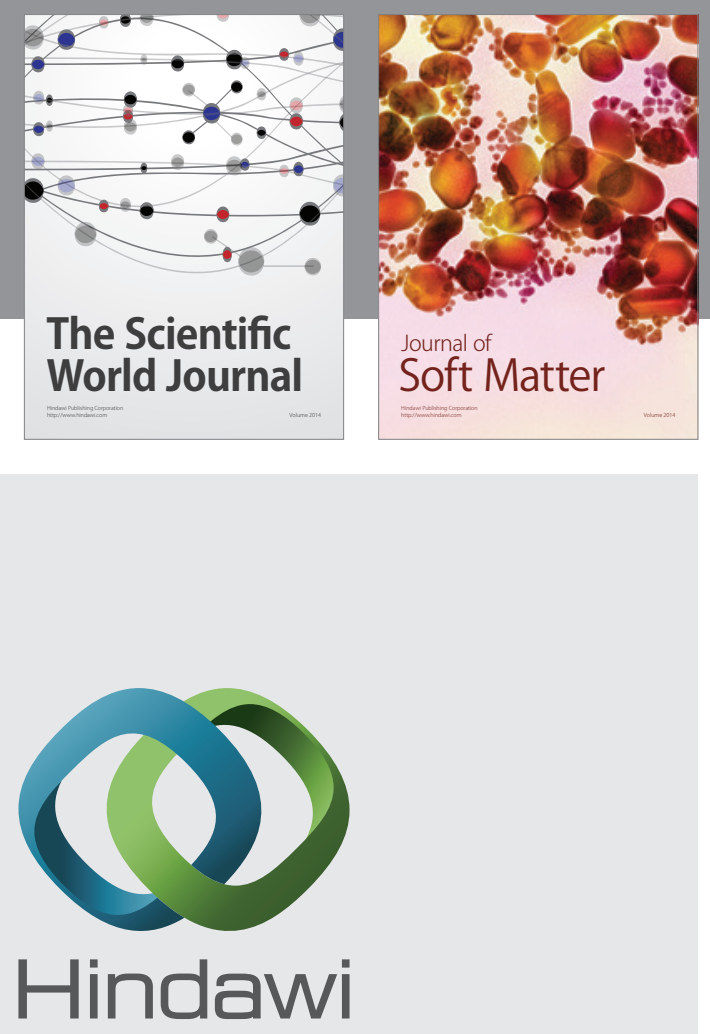

Submit your manuscripts at

http://www.hindawi.com
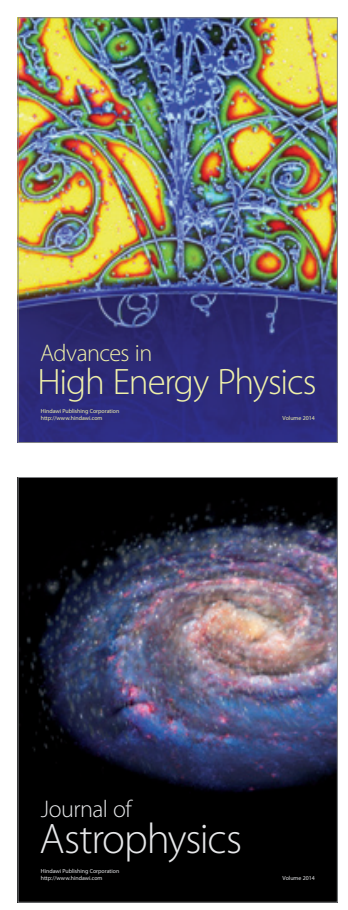
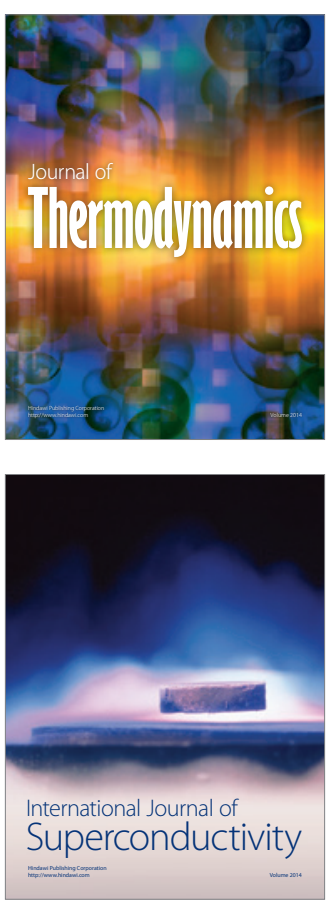
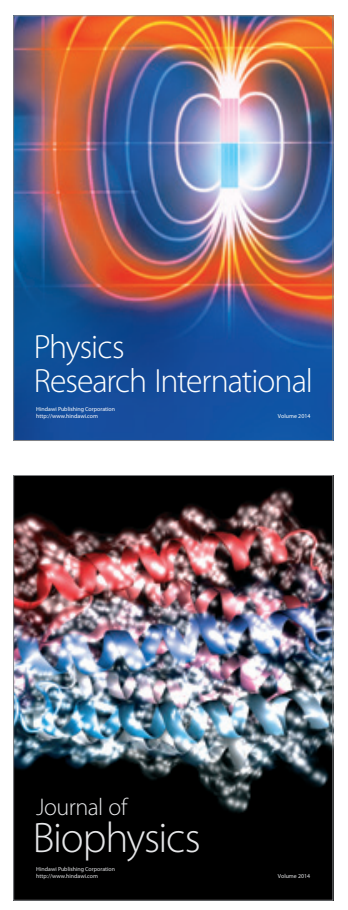
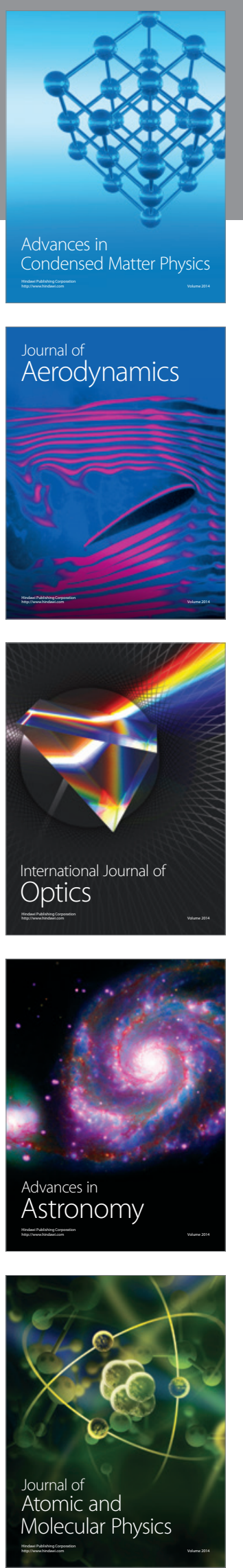